SIAC-PUB - $14 I$

October 1965

\title{
GROUP THEORY AND THE HYDROGEN ATOM (II)*
}

\author{
Myron Bander and Claude Itzyks on ${ }^{* *}$ \\ Stanford Iinear Accelerator Center \\ Stanford University, Stanford, California
}

\begin{abstract}
A previous work dealing with bound states in a Coulomb potential is extended to the case of scattering states. The symmetry of the problem under the Lorentz group $O(1,3)$ is used to construct wave functions. Harmonic analysis on two-sheeted hyperboloids is briefly discussed in arbitrary dimension. The set of scattering states for both an attractive and a repulsive potential is shown to provide a unitary representation of the group $O(1,4)$.
\end{abstract}

(To be submitted to Reviews of Modern Physics)

* Work supported by the U. S. Atomic Energy Commission

** On leave from Service de Physique Theorique, CEN Saclay, BP No. 2, Gif-sur-Yvette (Seine et Oise), France 


\section{INTRODUCTION}

In a previous article, hereafter denoted by $I$, we have reviewed the syrmetries of the Schroedinger equation for a Coulomb potential and discussed the use of a non-compact group isomorphic to the pseudo-orthogonal group $O(1,4)$ to relate the bound state levels. ${ }^{1}$ It is of some interest to pursue the analysis further to the scattering states. This is the goal of this work. The "hidden symmetry" is now an invariance under the homogeneous Lorentz group $O(1,3)$ and the space of scattering states can be written as a direct integral of infinite dimensional Hilbert spaces, which are carrier spaces of unitary representations of the Lorentz group. This is a frequent occurrence when dealing with a non-compact group, the simplest example being Fourier analysis for the group of translations in one dimension. We shall follow the usual device of introducing non-normalizable scattering states in order to achieve the decomposition. Physically, of course, the direct integral is related to the continuous spectrum of the Hamiltonian. As in the bound state case, there exists a larger group, isomorphic to $0(1,4)$ which connects the scattering states, but we have to introduce wave functions for both attractive and repulsive potentials. We shall show that the representation of this group, obtained in this way, is equivalent with the one previously discussed in $I$. This representation is in fact not unique and the ambiguity which arises is the same as the one aiready encountered.

We shall slightly generalize the discussion by taking an arbitrary dimension $f$ for the configuration space. In order to construct the wave functions it will be necessary to use harmonic analysis on a two-sheeted hyperboloid. This has been developed in four dimensions in a series of 
papers by Dolginov and collaborators ${ }^{2}$ but we shall briefly recall the main features including orthogonality and completeness of the "spherical functions." This part can perhaps be used for other purposes in a different context.

In an appendix we have performed the necessary transformations in order to show that the wave functions coincide with their ordinary expressions in configuration space, both for scattering and bound states.

We will have to use repeatedly the following notations:

$S_{p}$ : unit sphere in a p-dimensional real Euclidian space, the measure on the sphere being $\mathrm{d}^{\mathrm{p}-1} \Omega$ with

$$
\int_{S_{p}} d^{p-I_{\Omega}}=\omega_{p}=\frac{2 \pi^{p / 2}}{\Gamma(p / 2)}
$$

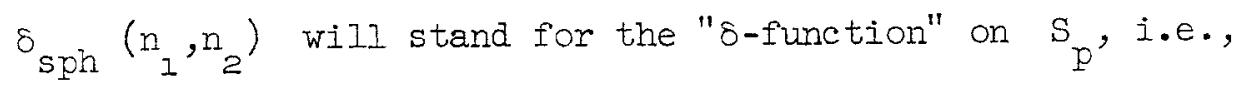

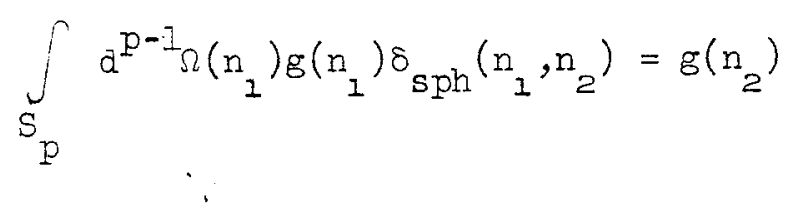

$T_{p}$ : unit hyperboloid in a $p$-dimensional real Euclidian space,

$$
u: u_{0}^{2}-\sum_{i=1}^{p-1} u_{i}^{2}=1
$$

$\mathrm{T}_{\mathrm{p}}^{ \pm}$will denote respectively the upper $\left(u_{0} \geq 1\right)$ or lower $\left(u_{0} \leq-1\right)$ sheet of this hyperboloid. Our constant parameterization of $\mathrm{T}_{\mathrm{p}}^{+}$ will be: $u_{0}=\operatorname{ch} \theta, u_{i}=\operatorname{sh} \theta n_{i}$, with $\theta \geq 0$ and $n_{i}$ on $s_{p-I}$. 
The measure on $T_{p}^{+}$will be written as $a^{p-1} \mu$ with $d^{p-1} \mu=\operatorname{sh} \theta^{p-2} d \theta d^{p-2}$. The measure on $T_{p}^{-}$will be related to the one on $\mathrm{T}_{\mathrm{p}}^{+}$by the transformation $u \in T_{p}^{-} \rightarrow u \in T_{p}^{+}$. Finally, $\delta_{h y p}\left(u_{1}, u_{2}\right)$ will stand for the " $\delta$-function" on $T_{p}^{+}$.

\section{THE SYMMETRY GROUP}

\section{Infinitesimal Method}

We study the scattering states in a Coulomb potential. As in I, we introduce the following two-vector operators:

the angular momentum,

$$
\vec{L}=\frac{1}{2}(\vec{r} \times \vec{p}-\vec{p} \times \vec{r})
$$

and the Runge-Lenz vector,

$$
\vec{M}=\frac{I}{2 \mu}(\vec{p} \times \vec{L}-\vec{L} \times \vec{p})-k \hat{r}
$$

with $\hat{r}$ standing for $\vec{r} /|\vec{r}| ; \vec{p}$ is the linear momentum, $\mu$ the reduced mass; $\mathrm{k}$ denotes the strength of the potential.

The Hamiltonian

$$
H=\frac{p^{2}}{2 \mu}-\frac{k}{|\vec{r}|}
$$

commutes with both these vectors. They satisfy also the following conmutation relations:

$$
\begin{gathered}
{\left[I_{i}, I_{j}\right]=i \not h \epsilon_{i j k} I_{k}, \quad\left[M_{i}, M_{j}\right]=-\frac{2 i \not h H}{\mu} \epsilon_{i j k} I_{k}} \\
{\left[I_{i}, M_{j}\right]=i \not h \epsilon_{i j k} M_{k}}
\end{gathered}
$$


Consider the subspace corresponding to the positive spectrum of $H$. In this subspace let $\widetilde{M}_{i}=\sqrt{\frac{\mu}{2 H}} M_{i}$ where the square root of $H$ is defined by the condition of positiveness. Then $I_{i}$ and $\tilde{M}_{i}$ build up the lie algebra of the homogeneous Lorentz group $O(1,3)$ (our notation implies that $o(p, q)$ is the pseudo-orthogonal real group leaving the metric with $p$ plus signs and $q$ minus signs invariant). In terms of $I$ and $\tilde{M}$ the Harniltonian reaas:

$$
H=-\frac{k^{2} \mu}{2}\left[I^{2}-\tilde{M}^{2}+\not \not^{2}\right]^{-1}
$$

The requirement that $H$ be Hermitian restricts $I$ and $\widetilde{M}$ to be likewise Hermitian, and hence we are interested in unitary representations of the Lorentz group. ${ }^{3}$ These representations are labeled by two numbers $\left(l_{0}, c\right)$ with $l_{0}$ a non-negative integer and $c$ pure imaginary, $c=$ ip (the principal series) or $l_{0}=0 \quad 0 \leq c \leq 1$ (the supplementary series). $\frac{I^{2}-\tilde{M}^{2}}{\not^{2}}$ is a Casimir operator equal for each representation to $l_{0}^{2}+c^{2}-1$. From Eq. (I) it follows that we have one further relation among $I$ and $\widetilde{M}$, name ly

$$
I \cdot \tilde{M}+\tilde{M} \cdot I=0=2 i l_{0} c
$$

Equation (3) thus restricts us to the representations with $l_{0}=0$. Thus

$$
E=-\frac{k^{2} \mu}{2 k^{2}} \cdot \frac{1}{c^{2}}
$$

where $E$ is the energy; and as we have assumed $H$ to be positive $c=i p$ and we are only concerned with representations of the principal series. 
2. Global Method - The Fock Transformation

As mentioned above, we shall now generalize the problem to an arbitrary dimension $f \geq 2$. The Schroedinger equation in momentum space takes the form

$$
\left(p^{2}-2 \mu E\right) \Phi(\vec{p})=\frac{2 \mu k}{\pi \omega_{f-1} \not h} \int a^{f} q \frac{\Phi(\vec{q})}{|\vec{p}-\vec{q}| f-I}
$$

with $\mathrm{E}>0$. Changing variables from $\overrightarrow{\mathrm{p}}$ to $(2 \mu \mathrm{E})^{-\frac{1}{2}} \overrightarrow{\mathrm{p}}$ and letting

$$
\Phi(\vec{p})=\Psi\left((2 \mu E)^{-\frac{1}{2}} \vec{p}\right)
$$

$\mathrm{Eq}$. (5) becomes

$$
\left(p^{2}-1\right) \Psi(\vec{p})=\frac{k(2 \mu)^{\frac{1}{2}}}{\pi \omega_{f-1} \not E^{\frac{1}{2}}} \int \vec{a}^{f} q \frac{d^{\Psi}(\vec{q})}{|\vec{p}-\vec{q}|^{f-1}} .
$$

We now imbed the $f$-dimensional space into one of $f+1$ dimensions and perform a projection of the original momentum space onto a two-sheeted hyperboloid, $T_{f+l}$ (the Fock transformation, see Fig. 1 ). Let $u$ be an arbitrary point in the $f+l$-dimensional space with component $u_{0}$ along the $f+I$ direction and $\vec{u}$ its ordinary projection in the original space. We introduce a Minkowski metric into this space, i.e., $u^{2}=u_{0}^{2}-\vec{u}^{2}$. The hyperboloid is given by the equation $u^{2}=I$ and the point $\vec{p}$ corresponds to a point on this hyperboloid with coordinates:

$$
\vec{p} \rightarrow u=\left(\frac{1+p^{2}}{1-p^{2}}, \frac{2 \vec{p}}{1-p^{2}}\right)
$$

The region $p^{2}<1$ is mapped on the upper sheet while $p^{2}>1$ is mapped 
on the lower one. We shall need the following relations. If $\vec{p}$ and $\vec{q}$ correspond to $u$ and $v$ respectively, then

$$
\begin{aligned}
|\vec{p}-\vec{q}|^{2} & =-\frac{(u-v)^{2}}{\left(1+u_{0}\right)\left(1+v_{0}\right)}=\frac{\mid(u-v)^{2}}{\left|1+u_{0}\right|\left|1+v_{0}\right|} \\
a^{f} p & =\frac{2 \delta\left(u^{2}-1\right) d^{f+1} u}{\left|1+u_{0}\right|^{f}}=\frac{d^{f} \mu(u)}{\left|I+u_{0}\right|^{f}},
\end{aligned}
$$

where we remind ourselves that all scalar products involving $u$ and $v$ must be taken with the Minkowski metric. For $u$ given by Eq. (7) and

$$
\hat{\Phi}(u)=\left|1+u_{0}\right|^{-\frac{f+1}{2}} \Psi(\vec{p})
$$

we obtain the following equation for $\hat{\Phi}$ :

$$
\hat{\Phi}(u)=\frac{k(2 \mu)^{\frac{1}{2}} \epsilon\left(u_{0}\right)}{2 \pi \omega_{f-1} k m^{\frac{1}{2}}} \int_{T_{f+1}} d^{f} \mu(v) \frac{\hat{\Phi}(v)}{\left|(u-v)^{2}\right| \frac{f-1}{2}}
$$

with $\epsilon\left(u_{0}\right)=+I$ if $u_{0}>I, \epsilon\left(u_{0}\right)=-I$ if $u_{0} \leq-I$. The above equation exhibits explicitly the invariance of the problem under the group of homogeneous, metric preserving, transformations in a $f+l$-dimensional Minkowski space, i.e., under the group $O(I, f)$.

Equation (9) is of the same type as the one obtained in I for bound states which was solved using the properties of spherical harmonics on the sphere. It will turn out that the solution, in the present case, can also be obtained by introducing a set of "spherical functions" on the 
hyperboloid. (The terminology is somehow misleading and hyperboiic functions would seem more appropriate; however, we stick to this name which is apparently of general use.) However, while the spherical harmonics are well known, the corresponding functions for the "Lorentz group" $O(I, f)$ enjoy less popularity. As mentioned in the introduction, they were studied in particular for $f=3$ by Dolginov and collaborators. ${ }^{2}$ The case of $f=2$ was also used in the context of Regge poles but dates back in the mathematical literature to Mehler. We shall for the moment interrupt our discussion of the Coulomb problem to give a description of these functions in order to apply them to the solution of Eq. (9). However, they certainly deserve some study for their own sake and, while exhibiting some results with lots of "E-functions," we shall be careful to present the proofs in such a way that they can, hopefully, be made rigorous.

\section{Definition of Spherical Functions on Hyperboloids}

Let $T_{f+1}^{+}$be the upper sheet, $u_{0} \geq 1$, of the hyperboloid $T_{f+I}$, u: $u_{0}^{2}-\sum_{i=1}^{f} u_{i}^{2}=1$. Given two points on $T_{f+1}^{+}, u_{1}$ and $u_{2}$, there always exists a transformation $\Lambda \in O^{(+,+)}(I, f)$ such that $u_{1}=\Lambda_{2} \cdot O^{(+,+)}(I, f)$ is the component of the identity of $O(I, f)$. The set of transformations which leave a point invariant is isomorphic to a proper rotation group $O^{(+)}(f)$ so that $T_{f+1}^{+} \approx o^{(+,+)}(1, f) / O^{(+)}(f)$. The measure $d^{f} \mu$ is invariant under $0^{(+,+)}(I, f)$. Hence the Hilbert space $f+1$ of square integrable functions defined on $\mathrm{T}_{f+1}^{+}$,

$$
\sum_{f+1}=\left\{g: \int_{T_{f+1}^{+}}|g|^{2} d^{f} \mu<\infty\right\},
$$


is the carrier space of a unitary representation of the non-compact group $o^{(+,+)}(1, f)$

$$
g \rightarrow U_{\Lambda} g \text { with } \quad\left(U_{\Lambda} g\right)(u)=g\left(\Lambda^{-1} u\right)
$$

This representation is not irreducible and decomposes into a direct integral of irreducible ones:

$$
U_{\Lambda}=\int_{0}^{\infty} d N U_{\Lambda}^{N} .
$$

Each $U_{\Lambda}^{N}$ is itself a unitary irreducible representation of the group but again the non-compactness results in the fact that it is infinite dimensional. Different $\mathrm{N}$ correspond to inequivalent representations. Correspondingly, the space $\mathrm{H}_{\mathrm{f}+1}$ will be split as a direct integral of infinite dimensional Hilbert spaces and any function in $/ / f+1$ will have a representation

$$
g(u)=\int_{\sigma}^{\infty} d N \sum_{\nu} g_{N, v}(u)
$$

where $v$ is a aiscrete index which distinguishes the components in the space of the representation $v_{\Lambda}^{N}$. The function $g_{\mathbb{N}, v}(u)$ will not belong to $/ f+1$ but will satisfy a certain partial differential equation in terms of the Casimir operator of the group. Having properly chosen the indices, $g_{\mathrm{N}, v}$ will be proportional to a spherical function. We shall now derive the equation satisfied by these functions. Let $u \equiv(c h \theta, \operatorname{sh} \overrightarrow{0})$, we shall investigate functions of the type

$$
\mathrm{H}_{\mathrm{N}, \alpha, \beta}^{(f+I)}(\mathrm{u})=\mathrm{Z}_{\mathrm{N}, \alpha}^{(f)}(\theta) \mathrm{Y}_{\alpha, \beta}^{(f)}(\overrightarrow{\mathrm{n}})
$$


where $Y_{\alpha, \beta}^{(f)}(\vec{n})$ is a spherical harmonic on the sphere $S_{f}$. The discrete index $\alpha$ takes the values $\alpha=0,1,2, \ldots$. If $\vec{x}=\overrightarrow{r n}$ is a point in an $f$-dimensional Euclidian space, $r^{\alpha} Y_{\alpha, \beta}(f)(\vec{n})$ is a homogeneous polynomial of degree $\alpha$ in the components of $\vec{x}$ and

$$
\Delta_{f}\left(\left(x \alpha_{\alpha, \beta}^{\alpha_{Y}(f)}(\vec{n})\right)=0\right.
$$

with the Laplace operator given in polar coordinates by

$$
\Delta_{f} \equiv \frac{\partial^{2}}{\partial r^{2}}+\frac{f-1}{r} \frac{\partial}{\partial r}-\frac{\alpha^{2}}{r^{2}}
$$

and $\mathcal{L}^{2}$ is the generalization of the angular momentum and operates only on the angular variables. Hence

$$
\mathcal{L}^{2} \underline{Y}_{\alpha, \beta}^{(f)}(\vec{n})=\alpha(\alpha+f-2) Y_{\alpha, \beta}^{(f)}(\vec{n})
$$

The index $\beta$ distinguishes the finitely many, linearly independent, solutions of this equation. Once normalized, the spherical harmonics satisfy:

$$
\begin{aligned}
& \sum_{\alpha, \beta} Y_{\alpha, \beta}^{(f)}\left(n_{1}\right) \overline{Y_{\alpha, \beta}^{(f)}\left(n_{2}\right)}=\delta_{s p h}\left(n_{1}, n_{2}\right) \\
& \int_{S_{f}} a^{f-I_{\Omega}}(n) Y_{\alpha_{1}, \beta_{1}}^{(I)}(n) \overline{Y_{\alpha_{2}, \beta_{2}}^{(f)}(n)}=\delta_{\alpha_{1}, \alpha_{2}} \delta_{\beta_{1}, \beta_{2}} .
\end{aligned}
$$

In $f+I$ dimensions we write the wave equation in the neighborhood of $\mathrm{T}_{f+1}^{+}$:

$$
\square_{i+1} \equiv \frac{\partial^{2}}{\partial w_{0}^{2}}-\sum_{i=1}^{i} \frac{\partial^{2}}{\partial w_{i}^{2}}=\frac{\partial^{2}}{\partial \rho^{2}}+\frac{f}{\rho} \frac{\partial}{\partial \rho}-\frac{1}{\rho^{2}}
$$


where is the Casimir operator on the hyperboloid and $\mathrm{w}_{0}=\rho \operatorname{ch} \theta$, $w_{i}=\rho \operatorname{sh} \theta n_{i}, \rho$ close to +1 . Then $\mathrm{H}_{N, \alpha, \beta}(u)$ is required to be an eigenfunction of $\bar{\sigma}$, or $\rho^{\lambda} \mathrm{H}_{N, \alpha, \beta}(u)$ defined in the neighborhood of $\mathrm{T}_{f+1}^{+}$ to be an homogeneous function of $w=\rho u$, of degree $\lambda$, with $\lambda=-\frac{f-1}{2}+i N$ (N will turn to be real) such that

$$
\square_{f+1}\left(\rho^{\lambda} H_{N, \alpha, \beta}(u)\right)=0
$$

The condition on $N$ will be obtained by requiring that $H_{N, \alpha, \beta}^{(f+I)}(u)$ be a continuous function of $\mathrm{T}_{f+1}^{+}$with the smallest possible growth at infinity. The above conditions provide for $\mathrm{Z}_{N, \alpha}(\theta)$ the following equation:

$$
\left\{\operatorname{sh}^{2} \theta \frac{d^{2}}{d \operatorname{ch} \theta^{2}}+f \operatorname{ch} \theta \frac{\alpha}{d \operatorname{ch} \theta}-\frac{\alpha(\alpha+f-2)}{\operatorname{sh}^{2} \theta}+N^{2}+\left(\frac{f-1}{2}\right)^{2} z_{N, \alpha}^{(f)}(\theta)=0\right.
$$

The boundary conditions just mentioned require $\mathbb{N}$ to be real and the relevant solutions of Eq. (15) are for our purpose - including a normalization to be discussed later:

$$
\begin{aligned}
& \text { for } \underbrace{f}_{f>3} \text { odd; } \\
& \mathrm{Z}_{N, \alpha}^{(f)}(\theta)=\left[\frac{\pi}{2} \cdot \mathrm{N}^{2}\left(\mathrm{~N}^{2}+1^{2}\right) \ldots\left(\mathrm{N}^{2}+(f-1+\alpha)^{2}\right)\right]^{-\frac{1}{2}} \times \operatorname{sh} \theta^{\alpha}\left(\frac{a}{\operatorname{dch} \theta}\right)^{\frac{f-1}{2}+\alpha} \cos N \theta \\
& \text { for } f \text { even; } \\
& f \geq 2 \\
& \mathrm{z}_{\mathrm{N}, \alpha}^{(f)}(\theta)=\left[\frac{\left(N^{2}+\left(\frac{1}{2}\right)^{2}\right)\left(N^{2}+\left(\frac{3}{2}\right)^{2}\right) \cdots\left(N^{2}+\left(\frac{f-2}{2}+\alpha-\frac{1}{2}\right)^{2}\right)}{N \text { th } \pi N}\right]^{-\frac{1}{2}} \times \operatorname{sh} \theta\left(\frac{\alpha}{\alpha \operatorname{ch} \theta}\right)^{\frac{f-2}{2}+\alpha} P_{i N-\frac{1}{2}}(\operatorname{ch} \theta)
\end{aligned}
$$

where it is understood in Eq. (17) that the factor on the right-hand side reduces to $(N \operatorname{th} \pi N)^{\frac{1}{2}}$ for $f=2 ; P_{i N-\frac{2}{2}}(\operatorname{ch} \theta)$ is the Iegendre function, and 
we shall throughout follow and use Chap. III of reference 4 for these functions which in this context are called conical functions. In both cases we have an even function of $\mathbb{N}$; in the following it is assumed that $N$ is positive. With $\mathrm{z}_{\mathrm{N}, \alpha}^{(f)}(\theta)$ given by Eq. (16) or (17), we have the following basic three relations for spherical functions:

Orthogonality relations, $N_{1}$ and $N_{2}$ are positive:

$$
\int_{T_{f+1}^{+}} d^{f} \mu(u) H_{N_{1}, \alpha_{1}, \beta_{1}}^{(f+1)}(u) \bar{H}_{N_{2}, \alpha_{2}, \beta_{2}}^{(f+1)}(u)=\delta_{\alpha_{1}, \alpha_{2}} \delta_{\beta_{1}, \beta_{2}} \delta\left(N_{1}-N_{2}\right) \text {, }
$$

completeness relations in $7 l_{f+1}$ :

$$
\int_{0}^{\infty} d N \sum_{\alpha, \beta} F_{N, \alpha, \beta}^{(f+I)}\left(u_{1}\right) H_{N, \alpha, b}^{(f+I)}\left(u_{2}\right)=\delta_{h y p}\left(u_{2}, u_{2}\right)
$$

integral equation

$\int_{f+1}^{+} d^{f} \mu(v) \frac{H_{N, \alpha, b}^{(f+I)}(v)}{\left(I+t^{2}+2 t u \cdot v\right)^{\frac{f-1}{2}}}=\frac{2 \pi^{\frac{f+I}{2}}}{\Gamma\left(\frac{f-I}{2}\right)} \cdot \frac{t^{-\frac{f-I}{2} \cos (N \log t)}}{N \sin \pi N} \cdot H_{N, \alpha, b}^{(f+I)}(u)$.

In Eq. (20) $t$ is a complex variable in a cut plane from - $\infty$ to 0 ; log $t$ is real for $t$ real positive and the argument of the expression $\left(1+t^{2}+2 t u \cdot v\right)$ which appears in the integrand is 0 for $t$ real positive. As is apparent from their definition [Eqs. (16) and (17)], the spherical functions are somehow different according to whether $f$ is even or odd. This again reflects the fact that the kernel in $\mathrm{Eq}$. (20) has a square root singularity for even $f$. Hence, we shall distinguish between the two cases to prove our basic three relations. 
4. Proof of the Three Relations in the Case $f$ Odd

We start with the orthogonality relations. According to Eqs. (II) and

(13) one has

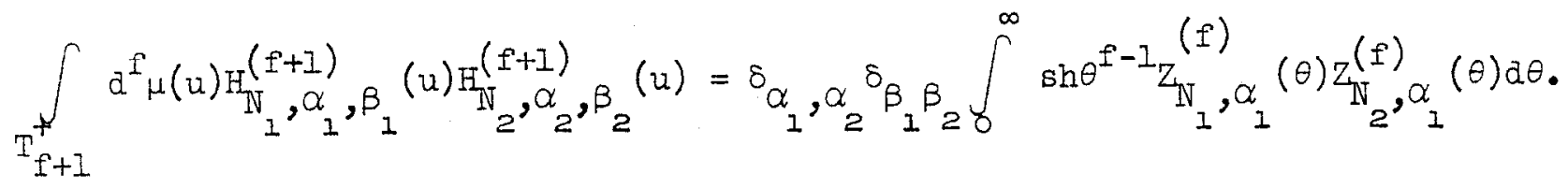
Using the definition of $\mathrm{Z}_{\mathbb{N}, \alpha}^{(f)}[\mathrm{Eq}$. (16)], this reduces to the study of the integral:

$$
\int_{0}^{\infty} a \theta \operatorname{sh} \theta^{f-1+2 \alpha_{1}}\left[\left(\frac{a}{d \operatorname{ch} \theta}\right)^{\frac{f-1}{2+\alpha}} \cos _{1} \theta\right]\left[\left(\frac{a}{\operatorname{den} \theta}\right)^{\frac{f-1}{2}+\alpha_{1}} \cos \pi_{2} \theta\right],
$$

Let us introduce the notation $q_{p}(N, \Omega)=\operatorname{sh} \theta^{p}\left(\frac{a}{\operatorname{dch} \theta}\right)^{p} \cos w \theta$. We want to evaluate

$$
\int_{0}^{\infty} d \theta q_{p}\left(N_{I}, \theta\right) q_{p}\left(N_{2}, \theta\right) .
$$

It is understood that $\mathbb{N}_{1}$ and $N_{2}$ are positive. The auxiliary functions $q_{p}(N, \theta)$ enjoy the following properties which are easily established:

$$
\begin{gathered}
q_{p+1}(N, \theta)=\left(\frac{a}{d \theta}-p \operatorname{cth} \theta\right) q_{p}(N, \theta) \\
\left(N^{2}+(p-1)^{2}\right) q_{p-1}(N, \theta)=-\left(\frac{d}{d \theta}+(p-1) \operatorname{cth} \theta\right) q_{p}(N, \theta) \\
\left\{\frac{d^{2}}{d \theta^{2}}+\mathbb{N}^{2}-\frac{p(p-1)}{\operatorname{sh}^{2} \theta}\right\} q_{p}(N, \theta)=0 .
\end{gathered}
$$


$q_{p}(N, 0)=0$ if $p \geq I$ and $q_{p}(N, \theta)$ has an oscillatory behavior at infinity. Using the recurrence relations given above we find by using integration by parts that for $p \geq I$ :

$$
\begin{aligned}
\int_{0}^{\infty} d \theta q_{p}\left(\mathbb{N}_{I}, \theta\right) q_{p}\left(N_{2}, \theta\right) & =\int_{0}^{\infty} d \theta q_{p}\left(\mathbb{N}_{I}, \theta\right)\left(\frac{d}{d \theta}-(p-I) \operatorname{cth} \theta\right) q_{p-I}\left(\mathbb{N}_{2}, \theta\right) \\
& =\int_{0}^{\infty} d \theta q_{p-1}\left(\mathbb{N}_{2}, \theta\right)\left(-\frac{d}{d \theta}-(p-I) \operatorname{cth} \theta\right) q_{p}\left(N_{I}, \theta\right) \\
& =\left[\mathbb{N}^{2}+(p-1)^{2}\right] \int_{\delta}^{\infty} d \theta q_{p-1}\left(\mathbb{N}_{1}, \theta\right) q_{p-I}\left(\mathbb{N}_{2}, \theta\right) .
\end{aligned}
$$

Since for $p=0, q_{p}(N, \theta)$ reduces to $\cos N \theta$ and

$$
\int_{0}^{\infty} d \theta \cos \mathbb{N}_{1} \theta \cos \mathbb{N}_{2} \theta=\frac{\pi}{2} \delta\left(\mathbb{N}_{1}-N_{2}\right)
$$

we find for $p \geq 1$

$$
\int_{0}^{\infty} d \theta q_{p}\left(N_{1}, \theta\right) q_{p}\left(N_{2}, \theta\right)=\left[N_{1}^{2}\left(N_{1}^{2}+I^{2}\right) \ldots\left(N_{1}^{2}+(p-I)^{2}\right] \frac{\pi}{2} \delta\left(N_{1}-N_{2}\right) .\right.
$$

Let now $p=\alpha_{1}+\frac{f-1}{2}$ and introduce the proper normalization for $z_{N}^{(f)} \alpha$. One sees that we have just proved Eq. (18).

We turn to the completeness relation. For that purpose we use the following addition theorem (see reference 4):

$$
\begin{aligned}
& \sum_{\alpha, \beta} H_{N, \alpha, \beta}^{(f+1)}\left(u_{1}\right) \frac{H_{N, \alpha, \beta}^{(f+1)}\left(u_{2}\right)}{(-1)^{\frac{f-1}{2}}}\left(\frac{a}{\frac{d}{\frac{f-1}{2}}}\right)^{\frac{f-1}{2}} \cos N \theta, \\
& f \text { off } \geq 3, \quad u_{1} \cdot u_{2}=\operatorname{ch} \theta .
\end{aligned}
$$


Integrating this result over $\mathbb{N}$ requires a little attention, since in interpreting the result as $\delta_{\text {hyp }}\left(u_{1}, u_{2}\right)$ we shall naturally want to adapt the coordinate system on the hyperboloid in order that, say, $u_{1}$ be the point $(1,0,0, \ldots, 0)$. Integration of $\mathrm{Eq}$. (2I) over $N$ will introduce "S-functions" of $\theta$. However, with this special system of coordinates, $\theta=0$ will be an end point of the integration interval in $\theta$. Hence we use the following procedure. We multiply Eq. (2I) by $\cos (\mathrm{N} \epsilon$ ), with $\epsilon>0$, $\epsilon$ will be allowed to go to zero at the end of the calculation (the same device will later be used without comment in the case of $f$ even). With this in mind we find:

$\int_{0}^{\infty} d N \sum_{\alpha, \beta} H_{N, \alpha, \beta}^{(f+1)}\left(u_{1}\right) \overline{H_{N, \alpha, \beta}^{(f+1)}\left(u_{2}\right)}=\lim _{\epsilon \rightarrow+0} \frac{(-1)^{\frac{f-1}{2}}}{(2 \pi)^{\frac{f-1}{2}}} \cdot\left(\frac{a}{a \operatorname{ch} \theta_{12}}\right)^{\frac{f-1}{2}} \delta\left(\theta_{12}-\epsilon\right)$.

The right-hand side does not appear at first sight to be equal to $\delta_{h y p}\left(u_{1}, u_{2}\right)$, but is indeed equal to it. To see that, recall that $a^{f} \mu=\operatorname{sh} \theta^{f-1} d \theta d^{f-1} \Omega$. Adapting the coordinate system as explained above, we compute with a test function $\psi$ :

$$
\begin{aligned}
\lim _{\epsilon \rightarrow+0} \frac{(-I)^{\frac{f-1}{2}}}{(2 \pi)^{\frac{f-1}{2}}} \int_{0}^{\infty} d \theta \int_{S_{f}}^{\infty} a^{f-I} \Omega(n) \operatorname{sh} \theta^{f-1} \psi(\theta, \vec{n})\left(\frac{a}{a \operatorname{ch} \theta}\right)^{\frac{f-1}{2}} \delta(\theta-\epsilon) \\
=\lim _{\epsilon \rightarrow+0} \frac{1}{2 \cdot(2 \pi)^{\frac{f-1}{2}}} \int_{0}^{\infty} d \theta \int_{f} a^{f-1} \Omega(n) \delta(\theta-\varepsilon) \operatorname{sh} \theta\left(\frac{a}{a \operatorname{ch} \theta}\right)^{\frac{f-1}{2}} \operatorname{sh} \theta^{f-2} \psi(\theta, \vec{n}) .
\end{aligned}
$$


We now use the fact that $\operatorname{sh} \theta\left(\frac{d}{d \operatorname{ch} \theta}\right)^{q} \operatorname{sh} \theta^{f-2}$ goes to zero for $\theta \rightarrow+0$ as long as $q<\frac{f-1}{2}$ (recall that $f$ is odd and greater or equal to 3 ), while

$$
\lim _{\theta \rightarrow+0} \operatorname{sh} \theta\left(\frac{d}{a \operatorname{ch} \theta}\right)^{\frac{f-1}{2}} \operatorname{sh} \theta^{f-2}=\frac{\Gamma(I-1)}{2^{\frac{f-1}{2}-I} \Gamma\left(\frac{f-1}{2}\right)}
$$

Our integral is thus $\psi(0)$ (which stands for $\psi(0, \vec{n}), \vec{n}$ arbitrary, the value being the same for all $\vec{n})$ times $\Gamma(f-I) /(4 \pi)^{\frac{f-1}{2}} \Gamma\left(\frac{f-1}{2}\right) \omega_{f}$ which is in fact equal to 1 , using the area of the sphere given in the introduction $2 \pi^{1 / 2} / \Gamma\left(\frac{f}{2}\right)$. In short, Eq. (19) is provea.

It remains to prove $\mathrm{Eq} \cdot(20)$. Let $t$ be for the moment a real positive variable with log $t$ real and compute the following absolutely convergent integral:

$\frac{I}{t} \int_{0}^{\infty} \frac{d N}{\operatorname{sh} \pi N} \cos (N \log t) \frac{\sin N \theta}{\sin \theta}=\frac{I}{2 \operatorname{sh} \theta t} \int_{0}^{\infty} \operatorname{dN}\left(\frac{\sin N(\theta+\log t)}{\operatorname{sh} \pi \mathbb{N}}+\frac{\sin N(\theta-\log t)}{\operatorname{sh} \pi N}\right)$ It is easy to show that

$$
\int_{-\infty}^{+\infty} d x \frac{\sin y x}{\sin x}=\pi \text { th } \pi \frac{y}{2}
$$

Hence our integral is equal to

$$
\frac{1}{4 t \operatorname{sh} \theta}\left\{\operatorname{th} \frac{\theta+\log t}{2}+\operatorname{th} \frac{\theta-\log t)}{2}\right\}=\frac{1}{1+t^{2}+2 t \operatorname{ch} \theta}
$$


The integral is absolutely convcrfent for complex $t$ as long as $t$ varies in a plane cut from $-\infty$ to 0 , such that log $t$ is real for real positive t. For these values of $t$ we get by analytic continuation:

$$
\frac{1}{1+t^{2}+2 t \operatorname{ch} \theta}=\frac{-I}{t} \int_{0}^{\infty} \frac{d N}{N \operatorname{sh} \pi N} \cos (N \log t) \frac{d}{d \operatorname{ch} \theta}(\cos N \theta)
$$

The addition theorem (21) together with Eq. (22) yields with the same restrictions on $t$

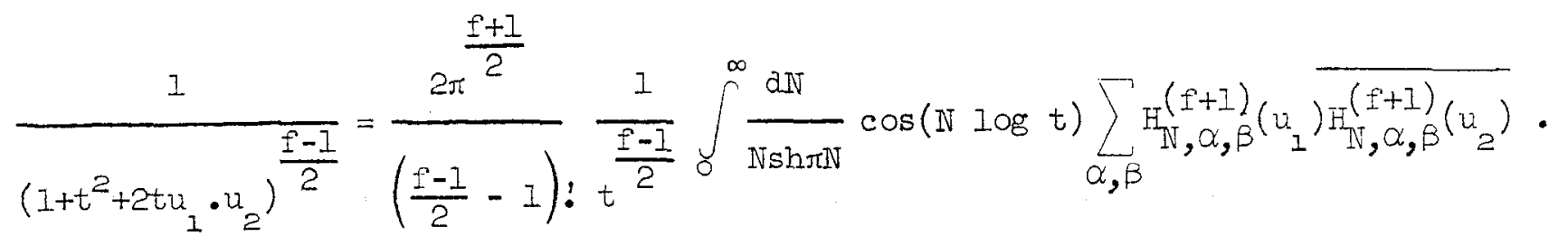

Hence, using the orthogonality relations Eq. (18), we get the desired result Eq. (20).

5. Proof of the Three Relations in the Case of $f$ Even

We first must give some properties of the Legendre functions which will be used in the following, principally the Mehler's transformation formulas. $\mathrm{P}_{\text {iN }-1 / 2}(\mathrm{z})$ is the solution of the Legendre equation:

$$
\left(\frac{a}{d z}\left(z^{2}-1\right) \frac{d}{d z}+N^{2}+\frac{1}{4}\right) P_{i N-I / 2}(z)=0
$$

with the property that it is regular at the point +1 where it takes the value +1 . For $z$ real greater than one, $\mathbb{N}$ real, which is the domain in which we are interested, $P_{i N-I / 2}$ is real and is even in $\mathbb{N}$. For $\theta$ real 
positive, the following two integral representations hold: ${ }^{4}$

$$
P_{i N-I / 2}(\operatorname{ch} \theta)=\frac{\sqrt{2}}{\pi \operatorname{th} \pi N} \int_{\theta}^{\infty} \frac{\sin N \psi d \psi}{(\operatorname{ch} \psi-\operatorname{ch} \theta)^{\frac{I}{2}}}=\frac{\sqrt{2}}{\pi} \int_{0}^{\theta} \frac{\cos N \psi}{(\operatorname{ch} \theta-\operatorname{ch} \psi)^{\frac{1}{2}}} d \psi
$$

These two integrals are reminiscent of the theory of Abel's integral equation. We propose to show that it leads us to the theory of Mehler's transforms. Let $g(\psi)$ be a function defined for $\psi \geq 0$ vanishing for $\psi=0$, square integrable between $O$ and $\infty$ and sufficiently regular for the following integrals to be well defined. One has the couple of equations:

$$
\begin{aligned}
& g(\psi)=\frac{2}{\pi} \int_{0}^{\infty} \sin \mathbb{N} \psi G(\mathbb{N}) d N \\
& G(N)=\int_{0}^{\infty} \sin N \psi g(\psi) d \psi .
\end{aligned}
$$

Consider the linear transformation:

$$
g(\psi) \rightarrow f(\theta)=\int_{0}^{\infty} \frac{g(\psi)}{(\operatorname{ch} \psi-\operatorname{ch} \theta)^{\frac{1}{2}}} d \psi
$$

where $\theta \geq 0$. The inverse formula of this Abel equation is defined, if say $g(\psi)<A \psi$ for small $\psi$, and gives us

$$
g(\psi)=-\frac{1}{\pi} \frac{a}{d \psi} \int_{\psi}^{\infty} \frac{f(\theta) \operatorname{sh} \theta d \theta}{(\operatorname{ch} \psi-\operatorname{ch} \theta)^{\frac{1}{2}}}
$$


Combining Eqs. (26) and (25) we find

$$
\begin{aligned}
f(\theta) & =-\frac{2}{\pi} \int_{0}^{\infty} d N G(N) \int_{\theta}^{\infty} \frac{\sin N \psi}{(\operatorname{ch} \psi-\operatorname{ch} \theta)^{\frac{1}{2}}} d \psi \\
& =\int_{0}^{\infty} d N P_{i N-I / 2}(\operatorname{ch} \theta) \sqrt{2}(\operatorname{th} \pi N) G(N)
\end{aligned}
$$

Where we have used the first integral representation in Eq. (24) for the Iegendre function. Similarly combining Eqs. (27) and (25) we tind:

$$
\begin{aligned}
G(N) & =\int_{0}^{\infty} d \psi(\sin N \psi) g(\psi)=-\int_{0}^{\infty} d \psi \sin N \psi-\frac{I}{\pi} \frac{d}{d \psi} \int_{\psi}^{\infty} \frac{f(\theta) \operatorname{sh} \theta}{(\operatorname{ch} \theta-\operatorname{ch} \psi)^{\frac{1}{2}}} d \theta \\
& =\left[-\frac{\sin \psi}{\pi} \int_{\psi}^{\infty} \frac{f(\theta) \operatorname{sh} \theta}{(\operatorname{ch} \theta-\operatorname{ch} \psi)^{\frac{1}{2}}} d \theta\right]_{0}^{\infty}+\frac{I}{\pi} \int_{0}^{\infty} \cdot d \psi N \cos N \psi \int_{\psi}^{\infty} \frac{f(\theta) \operatorname{sh} \theta}{(\operatorname{ch} \theta-\operatorname{ch} \psi)^{\frac{1}{2}}} d \theta
\end{aligned}
$$

Assuming that $g(\psi)$ is the derivative of a function which vanishes at infinity, we can drop the integrated term and we find, using the second integral representation in Eq. (24):

$$
G(N)=\frac{N}{2^{\frac{1}{2}}} \int_{0}^{\infty} \operatorname{sh} \theta \operatorname{def}(\theta) P_{i N-1 / 2}(\operatorname{ch} \theta) d \theta
$$

If we call $F(\mathbb{N})=G(\mathbb{N}) \sqrt{2}$ th $\pi \mathbb{N}$ we deduce from Eqs. (28) and (29) the couple of Mehler transforms:

$$
\begin{aligned}
& f(\theta)=\int_{0}^{\infty} F(N) P_{i N-I / 2}(\operatorname{ch} \theta) d N \\
& F(\mathbb{N})=\mathbb{N} \text { th } \pi \mathbb{N} \int_{0}^{\infty} d \theta \operatorname{sh} \theta f(\theta) P_{i N-I / 2}(\operatorname{ch} \theta) .
\end{aligned}
$$


We can express Eq. (30) in a different language with $x_{1}, x_{2}$ real and greater than one, $\mathbb{N}_{1}$ and $\mathbb{N}_{2}$ positive;

$$
\begin{aligned}
& \int_{0}^{\infty} d N P_{i N-I / 2}\left(x_{I}\right) P_{i N-I / 2}\left(x_{2}\right) N \text { th } \pi N=\delta\left(x_{I}-x_{2}\right) \\
& \int_{1}^{\infty} d x P_{i N_{1}-I / 2}(x) P_{i N_{2}-I / 2}(x)=\frac{\delta\left(N_{I}-N_{2}\right)}{N_{1} \text { th } \pi N_{1}}
\end{aligned}
$$

We are now in a position to derive formulas (18), (19) and (20) in the case of $f$ even $\geq 2$. From Eqs. (II) and (13) we get as before

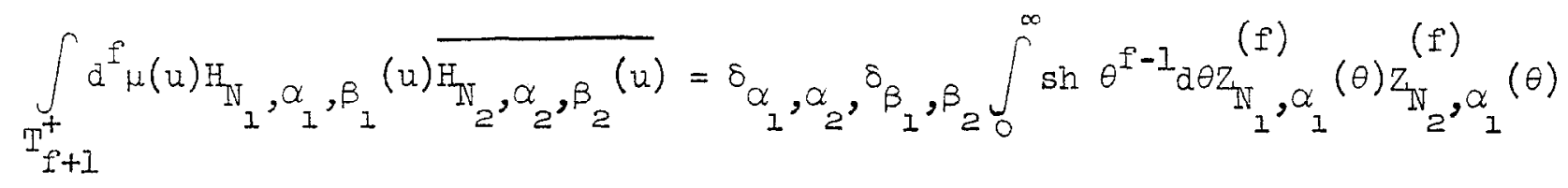
Using the definition of $\mathrm{Z}_{\mathrm{N}}^{(f)}, \alpha, \mathrm{Eq} .(17)$, leads us to study the integral:

$$
\int_{0}^{\infty} r_{\frac{f-2}{2}+\alpha}\left(N_{1}, \theta\right) r_{\frac{f-2}{2}+\alpha}\left(N_{2}, \theta\right) \operatorname{sh} \theta d \theta
$$

where the auxiliary function $r_{p}(N, \theta)$ (equal up to a factor to the associated Iegendre function) is defined through:

$$
r_{p}(N, \theta)=(\operatorname{sh} \theta)^{p}\left(\frac{d}{a \operatorname{ch} \theta}\right)^{p} P_{i N-1 / 2}(\operatorname{ch} \theta) .
$$

This function satisfies the following relations deduced from the analogous 
ones valia for the Legendre function:

$$
\begin{gathered}
r_{p+1}(N, \theta)=\left(\frac{d}{d \theta}-p \operatorname{cth} \theta\right) r_{p}(N, \theta) \\
\left(N^{2}+\left(p-\frac{1}{2}\right)^{2}\right) r_{p-1}(N, \theta)=-\left(\frac{d}{d \theta}+p \operatorname{cth} \theta\right) r_{p}(N, \theta) \\
{\left[\operatorname{sh}^{2} \theta\left(\frac{d}{d \operatorname{ch} \theta}\right)^{2}+2 \operatorname{ch} \theta \frac{d}{d \operatorname{ch} \theta}+N^{2}+\frac{1}{4}-\frac{p^{2}}{\operatorname{sh}^{2} \theta}\right] r_{p}(N, \theta)=0}
\end{gathered}
$$

Hence, by integration by parts using the fact that $r_{p}(N, \theta)$ is bounded at infinity by $A(\operatorname{ch} \theta)^{-\frac{1}{2}}$ :

$$
\begin{aligned}
\int_{0}^{\infty} d \theta \operatorname{sh} \theta r_{p}\left(\mathbb{N}_{I}, \theta\right) r_{p}\left(\mathbb{N}_{2}, \theta\right) & =\int_{0}^{\infty} d \theta \operatorname{sh} \theta r_{p}\left(N_{1}, \theta\right)\left(\frac{d}{d \theta}-(p-I) \operatorname{cth} \theta\right) r_{p-I}\left(\mathbb{N}_{2}, \theta\right) \\
& =-\int_{0}^{\infty} d \theta \operatorname{sh} \theta r_{p-1}\left(N_{2}, \theta\right)\left(\frac{d}{d \theta}+p \operatorname{cth} \theta\right) r_{p}\left(N_{1}, \theta\right) \\
& =\left[N^{2}+\left(p-\frac{I}{2}\right)^{2}\right] \int_{0}^{\infty} d \theta \operatorname{sh} \theta r_{p-I}\left(\mathbb{N}_{I}, \theta\right) r_{p-I}\left(\mathbb{N}_{2}, \theta\right) .
\end{aligned}
$$

Since for $p=0, r_{p}(N, 6)$ reauces to the Legendre function for which Eq. (3I) holds, we get

$\int_{0}^{\infty} d \theta \operatorname{sh} \theta r_{p}\left(\mathbb{N}_{I}, \theta\right) r_{p}\left(N_{2}, \theta\right)=\left[\left(\mathbb{N}_{I}^{2}+\left(p-\frac{1}{2}\right)^{2}\right) \ldots\left(N^{2}+\frac{1}{4}\right)\right] \frac{I}{N_{1} \operatorname{th} \pi N_{I}} \delta\left(N_{I}-N_{2}\right)$. The coefficient in front of the $\delta$-function is to be understood as $\left(\mathbb{N}_{1} \text { th } \pi \mathbb{N}_{1}\right)^{-1}$ if $p=0$. Comparing this result with the normalization of $\mathrm{z}_{\mathrm{N}, \alpha}^{(f)}(\theta)$ we see that we have proved Eq. (I8). 
For the completeness relations we use the addition therorem: ${ }^{4}$

$$
\begin{aligned}
& \sum_{\alpha, \beta} H_{N, \alpha, \beta}^{(f+1)}\left(u_{1}\right) \overline{H_{N, \alpha, \beta}^{(f+1)}\left(u_{2}\right)}=\frac{n \text {th } \pi N}{(2 \pi)^{f / 2}}(-1)^{\frac{f-2}{2}}\left(\frac{a}{a \operatorname{ch} \theta}\right)^{\frac{f-2}{2}} P_{i N-I / 2}(\operatorname{ch} \theta) . \\
& \operatorname{ch~} 0=\mu_{1} \cdot u_{2}
\end{aligned}
$$

To prove Eq. (19) it remains to integrate Eq. (32) over N. With the same trick as in the previous section, we find with the help of Eq. (3I):

$$
\int_{0}^{\infty} d N \sum_{\alpha, \beta} H_{N, \alpha, \beta}^{(f+1)}\left(u_{1}\right) \frac{H_{N, \alpha, \beta}^{(f+1)}\left(u_{2}\right)}{H_{1 \rightarrow+0}}=\lim _{\epsilon \rightarrow+1)^{\frac{f-2}{2}}} \frac{d}{(2 \pi)^{f / 2}}\left(\frac{f-2}{\alpha \operatorname{ch} \theta}\right)^{\frac{f}{2}} \delta(\operatorname{ch} \theta-1-\epsilon)
$$

Again, this is indeed $\delta_{\text {hyp }}\left(u_{1}, u_{2}\right)$ since with the test function $\psi$

$$
\lim _{\epsilon \rightarrow+0} \int_{0}^{\infty} a^{f} \mu(u) \psi(u) \frac{(-1)^{\frac{f-2}{2}}}{(2 \pi)^{f / 2}}\left(\frac{d}{a \operatorname{ch} \theta}\right)^{\frac{f-2}{2}} \delta(\operatorname{ch} \theta-I-\epsilon)
$$

$=\lim _{\epsilon \rightarrow+0} \int_{0}^{\infty} \operatorname{sh} \theta \operatorname{d\theta \delta }(\operatorname{ch} \theta-I-\epsilon) \frac{I}{(2 \pi)^{f / 2}}\left(\frac{a}{a \operatorname{ch} \theta}\right)^{\frac{f-2}{2}} \operatorname{sh} \theta^{\frac{f-2}{2}} \int_{S_{f}} \psi(\theta, \vec{n})^{f-1} d \Omega(n)$

Again

$$
\lim _{\theta \rightarrow+0}\left(\frac{a}{d \operatorname{ch} \theta}\right)^{q} \operatorname{sh} \theta^{f-2}=\left\{\begin{array}{l}
0 \text { if } q<\frac{f-2}{2} \\
\left(\frac{f-2}{2}\right) ! 2^{\frac{f-2}{2}} \text { if } q=\frac{f-2}{2}
\end{array}\right.
$$

so that the integral reduces by Leibniz rule to $\psi(0)$ times $\omega_{f}((f-2) / 2) ! 2^{(f-2) / 2}(2 \pi)^{f / 2}=1$. The completeness relations, Eq. (19), are thus proved. 
It remains to obtain the integral equation, (20). For this we use the alternative integral representation of $P_{i N-1 / 2}(\operatorname{ch} \theta)$ :

$$
P_{i N-I / 2}(\operatorname{ch} \theta)=\frac{\operatorname{ch} \tau \cdot N}{N} \int_{0}^{\infty} \frac{t^{-\frac{1}{2}-i N}}{\left(1+2 t \operatorname{ch} \theta+t^{2}\right)^{\frac{1}{2}}} d t .
$$

By Fourier transiormation Eq. (33) gives:

$$
t^{-\frac{1}{2}} \int_{0}^{\infty} \cos (N \log t) P_{i N-1 / 2}(\operatorname{ch} \theta) \frac{d N}{\operatorname{ch} \pi N}=\frac{1}{\left(1+2 t \operatorname{ch} \theta+t^{2}\right)^{\frac{1}{2}}} \cdot
$$

This result can again be continued analytically in the complex $t$ plane cut from $-\infty$ to 0 , with the principal determination of the logarithm in the left-hand side and the square root on the right-hand side such that it will be positive for real positive $t$.

Combining Eqs. (32) and (34) we get:

$\int_{0}^{\infty} \frac{d N}{N \operatorname{sh} \pi N} \cos (N \operatorname{Nog} t) \sum_{\alpha, \beta} H_{N, \alpha, \beta}^{(f+I)}\left(u_{1}\right) \overline{H_{N,}^{(f+I)}\left(u_{2}\right)}=\frac{t^{\frac{f-I}{2}}}{(2 \pi)^{f / 2}} \cdot \frac{1 \cdot 3 \ldots(f-3)}{\left(1+2 t u_{1} \cdot u_{2}+t^{2}\right)^{\frac{f-I}{2}}}$

with $1 \cdot 3 \ldots .(f-3)$ replaced by 1 if $f=2$ and the same restrictions on $t$ as before. With the help of the orthogonality relation, Eq. (18),

- we deduce from $\mathrm{Eq}$. (35) the desired integral equation, (20).

6. Solution of the Integral Equation for the Coulomb-Potential

Having now the required tools, we compare Eqs. (9) and (20). If we let $t$ go to 1 in Eq. (20) the expression is well defined. If we approach -1, either by the upper or lower imaginary plane, we again find a unique 
limit. Using these facts, let $g(u)$ be a function defined on the whole hyperboloid $T_{f+1}=T_{f+1}^{+}+T_{f+1}^{-}$, by

$$
\begin{aligned}
& u \in T_{f+1}^{+}, \quad g(u)=H_{N, \alpha, \beta}(u) \\
& u \in T_{f+1}^{-}, g(u)=a H_{N, \alpha, \beta}(-u) .
\end{aligned}
$$

One deduces from $E q .(20)$ that:

$\int_{T_{f+I}} a^{f} \mu(v) \frac{g(v)}{\left|(u-v)^{2}\right| \frac{f-I}{2}}=\frac{2 \pi^{\frac{f+I}{2}}}{\Gamma\left(\frac{f-I}{2}\right) N \operatorname{sh} \pi N} \begin{cases}a+\operatorname{ch} N \pi & \text { if } u \in T_{f+1}^{+} \\ \frac{I}{a}+\operatorname{ch} N \pi & \text { if } u \in T_{f+1}^{-}\end{cases}$

In order that $g(u)$ be a solution of $\mathrm{Eq} .(9)$, it is thus necessary that $(a+\operatorname{ch} \pi \mathbb{N})=-\left(\frac{1}{a}+\operatorname{ch} \pi \mathbb{N}\right)$, in which case $a=-e^{\bar{f} \pi \mathbb{N}} \cdot$ Correspondingiy we have two solutions (up to a normalization):

$$
\hat{\Phi}_{N, \alpha, \beta}^{( \pm)}(u)= \begin{cases}H_{N}, \alpha, \beta & \text { if } u \in T_{f+1}^{+} \\ -e^{\mp, N N} H_{N, \alpha, \beta}(-u) & \text { if } u \in T_{f+1}^{-}\end{cases}
$$

which satisfy

$$
\hat{\Phi}_{N, \alpha, \beta}^{( \pm)}(u)= \pm N \frac{\Gamma \frac{f-1}{2}}{\frac{f+1}{2}} \in(u) \int_{T_{f+1}} a^{f} \mu(v) \frac{\hat{\Phi}_{N, \alpha, \beta}^{ \pm}(v)}{\left|(u-v)^{2}\right| \frac{f-1}{2}} .
$$

Because of the completeness relations, Eq. (19), one can convince oneself that the functions, Eq. (36), exhaust the solutions of Eq. (9). The fact that for given $\mathbb{N}, \alpha, \beta$ there exist two solutions is a natural consequence from the fact that choosing $N$ positive was a matter of indifference; 
indeed $\hat{\Phi}_{N, \alpha, \beta}^{(+)}(u)=\hat{\Phi}_{-N, \alpha, \beta}^{(-)}(u)$. On the other hand, this double solution reflects the fact that we can as well treat attractive or repulsive potentials. $\hat{\Phi}(+)$ corresponds to the attractive case $(k>0), \hat{\Phi}^{(-)}$to the repulsive case $(k<0)$. Comparing Eqs. (9) and (37) we get the following relation between the energy $E$ and $\mathbb{N}$ :

$$
E=\frac{\mathrm{HK}^{2}}{2 k^{2}} \cdot \frac{7}{\mathrm{~N}^{2}}
$$

independently of the dimension $f$. One notes that Eq. (38) is the analytic continuation in the index $\lambda$ of the equation corresponding to bound states (Eq. (19) in I) from real integer values to complex values of the form $\lambda=-\frac{f-1}{2} i N$. So, in fact, are the eigenfunctions. We observe also in the case $f=3$, from the Iie-algebra analysis, that the eigenvalue $c$ which characterizes our representations of $O(I, 3)$ is equal to iN. Each set of functions $H_{N, \alpha, \beta}^{(f+1)}(u)$ for fixed $N$ provides a basis for an irreducible unitary representation of $0^{(+,+)}(1, f)$ through

$$
\mathrm{H}_{\mathrm{N}, \alpha, \beta}^{(f+1)}\left(\Lambda^{-1} u\right)=D_{\alpha^{\prime}, \beta^{\prime} ; \alpha, \beta^{N}}^{\mathrm{N}}(\Lambda) \mathrm{H}_{\mathrm{N}, \alpha^{\prime}, \beta^{\prime}}^{(f+1)}(u)
$$

This is clear since the equation satisfied by this function for fixed $\mathbb{N}$ is invariant under the group. We shall not exhibit explicitly the matrix elements of these representations; it would require too lengthy calculations. We return to this question in the following section.

\section{TRANSFORMATION GROUP}

We shall discuss briefly the introduction of a larger group of transformations. The motivation is the same as in I: We want to tind a group which relates the various scattering states corresponding to different 
energies but we have to mix solutions for attractive and repulsive potentials. Consider the space $f f_{f+1}$ of square integrable functions on $\mathrm{T}_{f+1}$ with the measure $d^{f} \mu$. It is spanned by our scattering states $\hat{\Phi}_{\mathbb{N}, \alpha, \beta}^{( \pm)}(u)$. We are looking for a group which contains $O(I, f)$ as subgroup and has a unitary representation in $f_{f+1}$ which reduces to the ones described above when restricted to $O\left(I, f^{\prime}\right)$. Again, the answer is in terms of projective transformations. We recall the construction of this group. Let us denote for the moment by $u$ an arbitrary point in Euclidian $f+I$ dimensional space. Introduce the quantity $z=u^{2}$ (where $u^{2}$ denotes the Minkowski square). In the $(z, u)$ space we are restricted to the previous "paraboloid." Consider the projective transformations which leave this parabolcid invariant. Introducing the homogeneity variable $t$ we find the homogeneous group, leaving

$$
z t-u^{2} \equiv\left(\frac{z+t}{2}\right)^{2}-\left(\frac{z-t}{2}\right)^{2}-u_{0}^{2}+\sum_{i=1}^{f} u_{i}^{2}
$$

invariant. That is the "conformal Lorentz group" $O(2, f+1)$. Going back to our $f+1$, Euclidian space we ask for the subgroup of $0(2, f+1)$ which leaves the initial hyperboloid $\mathrm{T}_{f+1}$ invariant. The condition is $z-t=0$. Hence the required group is $G \equiv O(1, f+1)$. The result turns out to be the same as for the bound state case. This will appear clear at the end of this section. Going through the previous transformations, the action of $G$ on the hyperboloid is found to be

$$
\begin{array}{r}
u \rightarrow \Lambda \cdot u=u^{\prime} ; \quad u, u^{\prime} \in T_{f+1} \\
u_{\alpha}^{\prime}=\frac{\sum_{\beta} a_{\alpha \beta} u_{\beta}+a_{\alpha t}}{\sum_{\beta} a_{t \beta} u_{\beta}+a_{t t}} .
\end{array}
$$


The Greek indices run from 0 to $f$, the Latin ones from $I$ to $f$. The real matrix $\Lambda$ belongs to $G$; that is

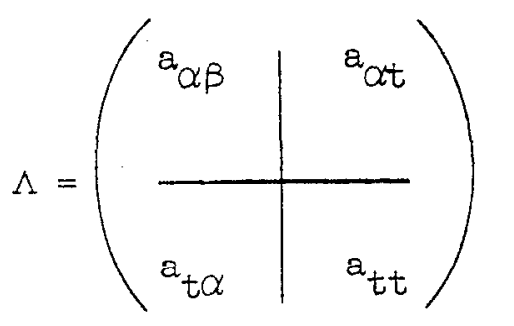

$$
\Lambda^{\mathrm{T}} \gamma \Lambda=\gamma
$$

The hyperboloid is clearly invariant since one finds

$$
u=1=\frac{u^{2}-1}{\left(\sum_{\alpha} a_{t \alpha} u_{\alpha}+a_{t t}\right)^{2}}=0
$$

It is to be remarked that in Eqs. (40) and (42) the denominator may vanish for certain transformations. This means in fact that in order to consider the action of $G$ on $T_{f+l}$ we have to add to the hyperboloid extra points "at infinity." In other words, $T_{f+I}$ has been compactified by the adjunction of a surface at infinity.

We build the following unitary representations of $G$ in $f+1$ which depend on the index $\rho$ :

$$
\Phi(u) \rightarrow \operatorname{T}_{\rho} \Phi(u)=\frac{\Phi\left(\Lambda^{-1} u\right)}{\left|\sum_{\alpha} a_{t \alpha}\left(\Lambda^{-1}\right) u_{\alpha}+a_{t t}\left(\Lambda^{-1}\right)\right|^{\frac{f}{2}+i p}} .
$$


Using Eq. (42) one checks the unitarity of these representations:

$$
\int_{T_{f+1}} d^{f} \mu \bar{\Phi} \psi=\int_{T_{f+1}} d^{f} \overline{\left[T_{\rho}^{\Lambda_{\Phi}}\right]}\left[\tilde{T}_{\rho} \psi\right]
$$

We have studied in I similar representations of $O(I, f+I)$ realized in the Hilbert space of square integrable functions on the sphere $\mathrm{S}_{f+1}$; denote it by $f_{f+1}$. It may be interesting to know whether we have constructed equivalent or inequivalent representations of the same group. The remainder of this section will be devoted to the proof of the unitary equivalence of the two sets of representations. All the properties investigated before, such as irreducibility, will thus hold true in the present case.

For that purpose we first define a transformation which maps the sphere

$$
s_{f+1}=\left\{v: v_{0}^{2}+\sum_{i=1}^{f} u_{i}^{2}=1\right\}
$$

on the hyperboloid

$$
T_{f+1}=\left\{u: u_{0}^{2}-\sum_{i=1}^{f} u_{1}^{2}=1\right\}
$$

It is the following mapping (see Fig. 2a)

$$
\begin{gathered}
v \in S_{f+1} \rightarrow u=\tau v \in T_{f+1} ; u=\left(\frac{I}{v_{0}}, \frac{-v_{i}}{v_{0}}\right) \\
u \in T_{f+1} \rightarrow v=\tau^{-i} u \in S_{f+1} ; v=\left(\frac{1}{u_{0}}, \frac{-u_{i}}{u_{0}}\right)
\end{gathered}
$$


Let $\mathrm{v} \rightarrow \Lambda \mathrm{v}=\mathrm{v}^{\prime}$ be a conformal transformation of $\mathrm{S}_{f+I} ; \Lambda \in O(I, f+I)$ and

$$
v_{0}^{\prime}=\frac{a_{00} v_{0}+\sum_{j}^{\prime} a_{0 j} v_{j}+a_{o t}}{a_{t 0_{0}}+\sum_{j} a_{t j} v_{j}+a_{t t}}, v_{i}^{\prime}=\frac{a_{i 0} v_{0}+\sum_{j} a_{i j} v_{j}+a_{i t}}{a_{t o} v_{0}+\sum_{j} a_{t j} v_{j}+a_{t t}}
$$

with

$$
\Lambda=\left(\begin{array}{l|l}
a_{t t} & a_{t \beta} \\
\hline a_{\alpha t} & a_{\alpha \beta}
\end{array}\right) ; \Lambda^{T} \gamma \Lambda=\gamma
$$

$\gamma$ being as in Eq. ( $\left.L_{1}\right)$. Performing the projection $T$ we find that the corresponding point on $\mathrm{T}_{f+l}$ undergoes the following transformation:

$$
\begin{aligned}
& u \rightarrow \Lambda_{T} u=u^{\prime} \\
& u_{0}=\frac{a_{t t u_{0}}-\sum_{j} a_{t j} u_{j}+a_{t 0}}{a_{o t} u_{0}-\sum_{j} a_{o j} u_{j}+a_{\infty}}, u_{i}=\frac{-a_{i t} u_{0}+\sum_{j} a_{i j} u_{j}-a_{i 0}}{a_{o t} u_{0}-\sum_{j} a_{0 j} u_{j}+a_{00}}
\end{aligned}
$$

That is with:

one has

$$
\Lambda_{T}=\left(\begin{array}{ccc}
I & 0 & 0 \\
0 & 0 & -I_{f} \\
0 & I & 0
\end{array}\right) \quad \Lambda_{0}^{-I}=\left(\begin{array}{ccc}
I & 0 & 0 \\
0 & 0 & I \\
0 & -I_{f} & 0
\end{array}\right)
$$

$$
\Lambda_{0} \Lambda_{0}^{-I}=\left(\begin{array}{ccc}
a_{t t} & -a_{t j} & a_{t o} \\
-a_{i t} & a_{i j} & -a_{i 0} \\
a_{c t} & -a_{0 j} & a_{00}
\end{array}\right)
$$


$I_{f}$ is a unit $f \times f$ matrix. The correspondence $\Lambda \rightarrow \Lambda_{\tau}$ is an inner automorphism of $O(I, f+1)$ and one even remarks that $\Lambda_{0}$ always belongs to the component of the identity.

If $u=T v$ one also finds, using Eq. (45), that

$$
a^{f} \Omega(v)=\frac{a^{f} \mu(u)}{\left|u_{0}\right|^{f}} ; u=\tau .
$$

Hence, if $\psi(v)$ is a function defined on $S_{f+1}$ and belong to $f i f$;

$$
\int_{S_{f+1}}|\psi(v)|^{2} d^{f} \Omega(v)=\int_{T_{f+1}} \frac{\left|\psi\left(\tau^{-1} u\right)\right|^{2}}{\left|u_{0}\right|^{f / 2}} d^{f} \mu(u) \quad:
$$

Using Eq. (5I) we define a unitary mapping $U_{\rho}$ from $h_{f+l}$ to $f_{f+1}$ by

$$
\begin{aligned}
& \psi(v) \in d l_{f+1}^{j} \rightarrow\left[U_{\rho} \psi\right](u)=\frac{\psi\left(\tau^{-I} u\right)}{\left|u_{0}\right|^{\frac{f}{2}+i \rho}} \in g b_{f+1} \\
& \Phi(u) \in \mathscr{H}_{f+1} \rightarrow\left[U_{\rho}^{-1} \Phi\right](v)=\frac{\Phi(T v)}{\left.\left.\right|_{v_{0}}\right|^{\frac{f}{2}+i \rho}} \in \psi_{f+1}
\end{aligned}
$$

The unitary representation of $O(I, f+1)$ that we investigated in I for $f_{f+1}$ was defined through

$$
\psi(v) \rightarrow\left[R_{\rho}^{\Lambda} \psi\right](v)=\frac{\psi\left(\Lambda^{-1} v\right)}{\left|\sum_{\alpha} a_{\epsilon \alpha}\left(\Lambda^{-1}\right) v_{\alpha}+a_{t t}\left(\Lambda^{-1}\right)\right|^{\frac{f}{2}+i \rho}}
$$


with $\Lambda \mathrm{v}$ given by Eq. (46). Similarly, Eq. (43) defines a unitary representation $\pi_{p}^{A}$ of the same group in $\mathscr{H}_{f+1}$. We will now prove that

$$
U_{\rho} R_{\rho}^{\Lambda}=T_{\rho}^{\Lambda_{T}} U_{\rho}
$$

Since in virtue of Eq. (49)

$$
T_{\rho}^{\Lambda_{T}}=T_{\rho}^{\Lambda_{0}} T_{\rho}^{\Lambda}\left(\begin{array}{c}
\Lambda_{0} \\
T_{p}
\end{array}\right)^{-1}
$$

and all operators are unitary, Eq. (54) is indeed a statement of unitary equivalence. The proof of this equality is rather straightforward. Consider for instance

$$
\left[U_{\rho} R_{\rho}^{\Lambda} \psi\right](u)=\frac{\psi\left(\Lambda^{-1} \tau^{-1} u\right)}{\left|a_{t t}\left(\Lambda^{-1}\right) u_{0}-\sum_{j} a_{t j}\left(\Lambda^{-1}\right) u_{j}+a_{t o}\left(\Lambda^{-1}\right)\right|^{\frac{\frac{f}{2}+i \rho}{2}}} ;
$$

on the other hand,

$$
\begin{aligned}
& {\left[T_{\rho}^{\Lambda} U_{\rho} \psi\right](u)=\frac{\psi\left(\tau^{-1} \Lambda_{\tau}^{-1} u\right)}{\left|a_{\infty 0}\left(\Lambda^{-1}\right) u_{0}-\sum_{j} a_{0 j}\left(\Lambda^{-1}\right) u_{j}+a_{o t}\left(\Lambda^{-1}\right)\right|^{\frac{f}{2}+i \rho}} x} \\
& \left.\left|\frac{a_{00}\left(\Lambda^{-1}\right) u_{0}-\sum_{j} a_{0 j}\left(\Lambda^{-1}\right) u_{j}+a_{0 t}\left(\Lambda^{-1}\right)}{a_{t t}\left(\Lambda^{-1}\right) u_{0}-\sum_{j} a_{t j}\left(\Lambda^{-1}\right) u_{j}+a_{t o}\left(\Lambda^{-1}\right)}\right|\right|^{\frac{\rho}{2}+i \rho}
\end{aligned}
$$


and since by definition of the automorphism $t, \tau^{-1} \Lambda_{T}^{-1}=\Lambda_{T}^{-1} \tau^{-1}$, Eq. (54) is easily obtained and the unitary equivalence established. The geometric transormation (45) which was at the basis of our proof is best understooa by adding an homogeneous coordinate such that $u_{0}=Y / T, u_{i}=X_{i} / T$ and the same for $\mathrm{v}$. The sphere and the hyperboloid appear as cones centered respectively around the $T$ and the $Y$ axis; the abstract group $O(I, \hat{I}+1)$ is realized in two different ways as homogeneous linear group which leave one of these cones invariant. A rotation of 180 degrees around the line $X_{i}=O, Y=T$ which is tangent to both cones is the substitute for the transformation $T$. This is pictured in Fig. 2-b.

As a matter of fact, one can prove that the unitary representations of $O(I, f)$ constructed in Section II with the heIp of spherical functions and those obtained in the section by means of conformal transformations on a sphere or an hyperboloid are equivalent. The proof is an extension of the one given in the case of $f=3$ in the third article of reference.

Before concluding this section we shall briefly mention the case of zero energy. From the commutation relations, Eq. (2), we note that $\left[\mathrm{M}_{\dot{i}}, \mathrm{M}_{j}\right]=0$ and the operators $\overrightarrow{\mathrm{I}}, \overrightarrow{\mathrm{M}}$, build the algebra of the Euclidian group in three dimensions, $E(3)$. In $f$ dimensions this may likewise be realized globally. We project the $f$ dimensional momentum space onto a paraboloid in $f+1$ dimensions, $P_{f+I}$. If $u$ is a point on the paraboloid $u_{0}=\vec{u}^{2} / 2$, with $u_{0}$ in the $f+1$ direction and $\vec{u}$ in the original $f$ dimensional subspace, we let $p \rightarrow u=\left[l /\left(2 p^{2}\right), \vec{p} / p^{2}\right]$. Performing this transformation on $\mathrm{Eq} \cdot(5)$ we obtain, with $\hat{\Phi}(u)=\Phi\left(\vec{p} / p^{2}\right) I / p^{f+1}$.

$$
\hat{\Phi}(u)=\frac{2 u k}{\pi \omega_{f-1} \not h} \int \frac{a^{f+1} f \delta\left(v_{0}-\frac{1}{2} \vec{v}^{2}\right) \hat{\Phi}(v)}{|\vec{u}-\vec{v}|^{f-1}} \text {, }
$$


which exibits the invariance of the problem under the Euclidian group in $f$ dimensions, $E_{f^{*}}$. It may also be noted that the group of conformal transformations in $f+l$ dimensions which leaves $P_{f+l}$ invariant is again $O(1, f+1)$. The action of the group on functions defined on $P_{f+1}$ may be obtained from its action on $S_{f+1}$ or $\mathrm{T}_{f+1}$ by noting that in Fig. 2-b, if we make a rotation of $45^{\circ}$ in the $(T, Y)$ plane, we transform $S_{f+1}$ and $\mathrm{T}_{f+1}$ into $\mathrm{P}_{f+1}$. It is amusing to note that the large group of transformations in all cases is $O(I, f+I)$ and the subgroups which are symetries of the problem in the cases $E<O, E=O, E>0$ are $O(f+I), E(f), O(1, f)$ respectively, which are the little groups of the Poincaré group in an $(I, f+l)$ dimensional Minhowski space.

\section{CONCLUUSION}

The hyörogen atom illustrates several aspects of the use of group theory, and especially non-compact groups, in quantum mechanics. The theory of infinite dimensional representations can be of interest in various problems. We hope to have shown in this work that it can be used beyond the realm of Lie algebras.

It is a pleasure to thank Professor Panofsky for his hospitality at SIAC. Thanks are due Dr. R. Stora for discussions on the necessity of including both attractive and repulsive solutions. 


\section{APPEINDIX}

In Section II, Eq. (36), and in Reference 1, Eqs. (12) and̄ (40), we obtained representations of the wave function in momentum spaces, in the scattering and bound-state cases, respectively. We shall explicitly transform back to configuration space and show that we obtain the usual expressions. We set, of course, $f=3$.

\section{Bound States}

Using the results of $I$, we find for the radial wave fun vtion with principal quantum number $n$ and angular momentum $l$ :

$$
\begin{aligned}
& \psi_{n, l}(r)=\frac{(-1)^{l} 8 p_{0}^{3 / 2}}{\pi h^{3 / 2}} \cdot\left[\left(n^{2}-1^{2}\right) \ldots\left(n^{2}-l^{2}\right)\right]^{-\frac{1}{2}} \int_{0}^{\infty} \frac{q^{2} d q}{\left(1+q^{2}\right)^{2}} \\
& \quad \cdot j l\left(q \frac{r p_{0}}{\not h}\right) \sin \delta^{l}\left(\frac{a}{a \cos \delta}\right)^{l} \frac{\sin n \delta}{\sin \delta} \\
& q=\operatorname{th} \delta / 2,
\end{aligned}
$$

where $p_{0}=\sqrt{-2 \mu E}$. We want to evaluate the last integral; denote it by $I_{n, l}\left(r p_{0} / h\right)$. It is clear that $I_{n, l}(y)_{y \rightarrow 0}=O\left(y^{l}\right)$. We consider the following sum for $|t|<1$

$$
\sum_{1}^{\infty} n I_{n, l}(y) t^{n}=\left(1-t^{2}\right) t^{l+1} 2^{2 \ell}(l+1): \int_{0}^{\infty} d q j l(q y)\left[\frac{q}{q^{2}(1+t)^{2}+(1-t)^{2}}\right]^{l+2} .
$$

The integrand is even; we can extend the integration from $-\infty$ to $+\infty$ ana use $j_{\ell}(z)=\frac{1}{2 i}\left\{h_{\ell}^{(+)}(z)-h_{\ell}^{(-)}(z)\right\}$. The Hankel functions satisfy: ${ }^{5}$

$$
h_{l}^{(+)}(-z)=(-1)^{l+1} h^{(-I)}(z), h_{l}^{( \pm)}(z)_{z \rightarrow 0}=O\left(z^{-l-1}\right) .
$$


Hence we can write:

$$
\begin{aligned}
\sum_{n} n I_{n}(y) t^{n} & =\frac{\left(1-t^{2}\right)}{2 i} t^{l+1} 2^{2 l}(l+1) ! \int_{-\infty}^{+\infty} d q h^{(t)}(q y)\left(\frac{q}{q^{2}(1+t)^{2}+(1-t)^{2}}\right)^{l+2} \\
& \left.=\frac{\left(1-t^{2}\right)}{2 i} t^{l+1} 2^{2 l} \frac{2 \pi i}{(1+t)^{l+2}}\left(\frac{d}{d q}\right)^{l+1} h_{l}^{(+)}(q y)\left(\frac{q}{q(1+t)+i(I-t)}\right)^{l+2}\right\}_{\alpha=i \frac{1-t}{1+t}}
\end{aligned}
$$

where the integral was performed by closing the contour in the upper half $q-p l a n e$. Since the sum behaves like $y^{l}$ for $y \rightarrow 0$ we can drop in the derivative all the terms which are of smaller power in $\mathrm{y}$. We use explicit form of the Hankel function ${ }^{5}$ and obtain:

$$
\sum_{1}^{\infty} n I_{n}, l^{(y)} t^{n}=\pi t^{l+I} 2^{l} \geq y^{l} e^{-y} \frac{e^{y \frac{2 t}{I+t}}}{(I+t)^{2 l+2}}
$$

On the right-hand side we recognize the generating function of associated Laguerre polynomials:

$$
\frac{e^{y \frac{t}{1+t}}}{(1+t)^{1+k}}=\sum_{p=0}^{\infty} \frac{(-1)^{p_{t}^{p}}}{(p+k) !} I_{p}^{k}(y) \quad|t|<1
$$

and thus get

$$
\begin{aligned}
I_{n, l}(y) & =\int_{0}^{\infty} \frac{q^{2} d q}{\left(1+q^{2}\right)} j_{l}(q y) \sin \delta^{l}\left(\frac{a}{a \cos \delta}\right)^{l}\left(\frac{\sin n \delta}{\sin \delta}\right)_{\delta=2 \operatorname{arct}} \\
& =\frac{(-1)^{n-l-1}}{n(n+l) ! r} \pi 2^{l-2} y^{l} e^{-y} L_{n-1}^{2 l+1}(2 y)
\end{aligned}
$$


Introducing the Bohr radius:

$$
a=\frac{\hbar^{2}}{k \mu}
$$

we obtain by combining Eqs. (A.I) and (A.2) the radial wave function:

$$
\left.\psi_{n, l}(r)=\frac{(-1)^{n-1}}{a^{3 / 2}} \cdot \frac{2}{n^{2}} \cdot\left[\frac{(n-l-1) !}{[(n+l) !]^{3}}\right]^{\frac{1}{2}} \cdot \rho^{l} e^{-\rho / 2} I_{n-l-1}^{2 l+1}(\rho) ; \rho=\frac{2 r}{n a} \quad \text { (A. } 4\right)
$$

\section{Scattering Case}

With the necessary adjustments for normalization we find for the radial wave function with angular momentum $l$ and energy

$$
\begin{aligned}
\Psi_{N, l}(r)= & \frac{4 \pi i^{l}}{(2 \pi)^{3 / 2}}\left\{\frac{N}{\left(1-e^{-2 \pi N}\right) N^{2}\left(N^{2}+I^{2}\right)\left(N^{2}+l^{2}\right)}\right\}^{\frac{1}{2}} \times \\
& {\left[\int_{0} d q q^{2} j_{l}(q \rho)\left(\frac{2}{1-q^{2}}\right)^{2} \sin \theta^{l}-\left(\frac{d}{d \operatorname{ch} \theta}\right)^{l+I} \cos N \theta_{-}\right.}
\end{aligned}
$$

$$
\left.-e^{-\pi \mathbb{N}} \int_{1}^{\infty} d q q^{2} j_{\ell}(q \rho)\left(\frac{2}{1-q^{2}}\right)^{2} \sin \theta_{+}^{\ell}\left(\frac{a}{d \operatorname{ch} \theta_{+}}\right)^{\ell+1} \cos N \theta_{+}\right]
$$

$N=k \frac{\mu}{2 h^{2} \mathrm{E}}, \rho=\frac{\sqrt{2 \mu \mathrm{E}}}{\not h} \mathrm{r}, \operatorname{th}\left(\theta_{-} / 2\right)=q$ for $0<q<1, \operatorname{th}\left(\theta_{+} / 2\right)=\frac{1}{q}$ for $q>1$.

The positive (negative) values of $N$ correspond to attractive (repulsive) potentials. Of course we must give some prescription to deal with the singular point $q=1$. We introduce

$$
F_{l}(\mathbb{N}, \theta)=-\frac{1}{\operatorname{sh} \pi \mathbb{N}}\left(\frac{d}{d \operatorname{ch} \theta}\right)^{l+1} \frac{\cos N \theta}{\operatorname{sh} \theta}
$$




$$
\begin{gathered}
\tilde{F}_{l}(t, \theta)=\int_{-\infty}^{+\infty} d N e^{i N t} F_{\ell}(\mathbb{N}, \theta)=\frac{1 \operatorname{sh} t(-1)^{l}(l+1) !}{(\operatorname{ch} \theta+\operatorname{ch} t)^{l+2}} \\
\psi_{\mathbb{N}, \ell}=\frac{4 \pi i^{l-1}}{(2 \pi)^{5 / 2}} \operatorname{sh} \pi \mathbb{N}\left[\frac{N}{\left(1-e^{-2 \pi N}\right) N^{2}\left(N^{2}+1^{2}\right) \ldots\left(N^{2}+l^{2}\right)}\right]^{\frac{1}{2}}(-1)^{l}(l+1) ! 2^{l+1} x \\
\int_{-\infty}^{+\infty} d t e^{-i N t} \operatorname{sht} \int_{-\infty}^{+\infty} d q j \ell(q \rho)\left(\frac{q}{q^{2}[1-\operatorname{ch}(t-i \epsilon)]+[1+\operatorname{ch}(t-i \epsilon)]}\right)^{l+2}
\end{gathered}
$$

The prescription $c h(t-i \epsilon)$ takes care of the singularity at $q=1$. We replace again $j_{l}$ by $\frac{1}{2 i}\left(h_{l}^{(+)}-h_{l}^{(-)}\right)$, close the contour in the upper half of the q-plane and use the explicit form of the Hankel function to calculate the derivative which occurs at the point $q=\operatorname{cth}\left(\frac{t-i \epsilon}{2}\right)$. The result is

$$
\psi_{N, l}=\frac{8 \pi^{2} i l}{(2 \pi)^{5 / 2}} \operatorname{sh} \pi\left[\frac{N}{\left(1-e^{-2 \pi \mathbb{N}}\right) N^{2}\left(N^{2}+1^{2}\right) \ldots\left(N^{2}+l^{2}\right)}\right]^{\frac{1}{2}} 2^{-l-2} \rho^{l} \int_{-\infty}^{+\infty} \frac{d t}{\left(\operatorname{sh}^{2} \frac{t}{2}\right)^{l+1}} e^{i c h \frac{t}{2} \cdot p-i N \pi t}
$$

We take $x=x \operatorname{th} \frac{t}{2}$ as a variable, the region of integration being the real axis except the segment $(-1,+1)$. We can close the contour in the upper half plane ( $\rho$ is positive) and move it to $(-1,+1)$. Taking into account changes of arguments in the integrand we get

$$
\psi_{\mathbb{N}, l}=\operatorname{sh} \pi \mathbb{N} \sqrt{\frac{\mathbb{N}}{2 \pi I-e^{-2 \pi N}}}\left(\frac{-i \rho}{2}\right)^{l} \frac{e^{\pi N}}{\sqrt{N^{2} \ldots\left(N^{2}+l^{2}\right)}} \int_{-1}^{+1} d x e^{i \rho x}(1-x)^{l-i N}(x+I)^{l+i N}
$$

with $\operatorname{Arg}(x+1)=\operatorname{Arg}(1-x)=0$. Apert from a proportionality factor and an 
exponential, the integrand is a classical representation of the hypergeometric function. Taking this factor into account and

$$
\sqrt{\pi N^{2}\left(N^{2}+1\right) \ldots\left(N^{2}+l^{2}\right)}=\sqrt{N \operatorname{sh} \pi N}|\Gamma(\ell+i N+1)|
$$

our result reads

$$
\psi_{N, l}(r)=e^{\frac{\pi N}{2}} \frac{|\Gamma(l+i N+1)|}{(2 l+1) !}\left(\frac{2 p}{i}\right)^{l} e^{-i p} F(l+i N+1 \mid 2 l+2 ! 2 i p) \quad \text { (A.6) }
$$

with $\rho$ as in $(A .5)$ 
LIST OF REFERENCES

1. M. Bander and C. Itzykson, "Group theory and the hydrogen atom," SLAC-FUB-120 (1965).

2. A. Z. Dolginov, "Relativistic spherical functions," JETP 3, 589 (I956); A. Z. Dolginov and I. N. Toptygin, "Relativistic spherical functions, II," JEIP 37, $1022(1960)$;

A. Z. Dolginov and A. N. Möskalev, "Relativistic spherical functions, III," JETP 37, 1202 (1960).

3. M. A. Naimark, Linear Representations of the Lorentz Group, American Mathematical Society Translations (1957), Series 2, Vol. 6.

4. Erdely, Magnus, Oberhettinger and Tricomi, Higher Transcendental Functions (McGraw-Hill Book Company, Inc., New York, 1953), Vol. I.

5. A. Messiah, Mécanique Quantique (Dunod, Paris, 1959), Tome 1. 